\title{
Interleukin-10 polymorphism in position -1082 and acute respiratory distress syndrome
}

\author{
M.N. Gong*, B.T. Thompson\# , P.L. Williams", W. Zhou', M.Z. Wang ${ }^{+}$, \\ L. Pothier ${ }^{+}$and D.C. Christiani ${ }^{\#,+}$
}

ABSTRACT: The GG genotype of the interleukin (IL)-10 promoter polymorphism in position -1082 (-1082GG) has been associated with increased IL-10 production. The current authors hypothesised that the $-1082 \mathrm{GG}$ genotype is associated with the development of, and outcomes in, acute respiratory distress syndrome (ARDS).

A nested case-control study was conducted in 211 Caucasian cases of ARDS and 429 controls who were admitted to an intensive care unit with sepsis, trauma, aspiration or massive transfusions. Cases were followed for organ failure and 60-day mortality.

The -1082GG genotype was associated with the development of ARDS, but only in the presence of a significant interaction between the -1082GG genotype and age. Among patients with ARDS, the -1082GG genotype was associated with decreased severity of illness on admission, lower daily organ dysfunction scores and lower 60-day mortality.

In conclusion, the high interleukin-10-producing -1082GG genotype may be associated with variable odds for acute respiratory distress syndrome development depending on age. Among those with acute respiratory distress syndrome, the -1082GG genotype is associated with lower mortality and organ failure. Further studies are needed to confirm these findings.

\section{KEYWORDS: Acute respiratory distress syndrome, interleukin-10, mortality, polymorphism}

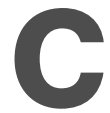
urrent understanding of why some individuals develop and die from acute respiratory distress syndrome (ARDS) while others do not is incomplete. Although clinical risks such as sepsis and trauma are well recognised, only a minority of patients with these risks develop ARDS [1]. Genetic polymorphisms important in innate immunity, pulmonary defence and inflammatory response have been found to be associated with the development of and outcomes in ARDS, sepsis and pneumonia [2-5], suggesting that genetic variation may explain some of the observed inter-individual differences in risk and outcomes in critical illnesses, such as ARDS.

Interleukin (IL)-10 is an important anti-inflammatory cytokine that modulates pro-inflammatory cytokines, such as tumour necrosis factor (TNF)- $\alpha$, as well as synthesis of nitric oxide, apoptosis of inflammatory cells and suppression of macrophage activation [6]. IL-10 attenuates the pro-inflammatory response in sepsis and reduces

For editorial comments see page 669. mortality in some animal models $[7,8]$, but not in others [9]. In humans, elevated circulating IL-10 has been associated with septic shock [10], severity of injury [11, 12] and mortality [13, 14]. Among patients with ARDS, the studies have been mixed. Lower levels of IL-10 were found in patients with ARDS compared with critically ill non-ARDS patients [15]. Among ARDS patients, high plasma IL-10 but low bronchoalveolar lavage concentration of IL-10 correlated with increased mortality $[16,17]$.

It has been reported that $50-75 \%$ of the variation in IL-10 production is genetically controlled [18, 19]. In the $I L-10$ gene, a $G$ to A single nucleotide promoter polymorphism at position -1082 is important in the regulation of IL-10. Individuals homozygous for the $G$ allele $(-1082 G G)$ have higher circulating IL-10, higher expression of IL10 mRNA, and greater production of IL-10 after in vitro stimulation [20-22].

Results from studies of the $I L-10-1082 G A$ polymorphism have been inconsistent and appear to depend on whether the study population and the controls are critically ill. In community-acquired

\section{AFFILIATIONS}

*Division of Pulmonary and Critical Care Medicine, Dept of Medicine, Mount Sinai School of Medicine, New York, NY, and

\#Pulmonary and Critical Care Unit, Dept of Medicine, Massachusetts General Hospital, Harvard Medical School, and

'Dept of Biostatistics, Harvard School of Public Health, and +Environmental Health Dept, Harvard School of Public Health, Boston, MA, USA.

\section{CORRESPONDENCE}

D.C. Christiani

Harvard School of Public Health

665 Huntington Avenue

Boston

Massachusetts 02115

USA

Fax: 16174323441

E-mail: dchristi@hsph.harvard.edu

Received:

April 182005

Accepted after revision:

November 152005

SUPPORT STATEMENT

The following work was supported by research grant R01 HL60710 from the National Heart, Lung and Blood Institute (NHLBI; Bethesda, MD,

USA). M.N. Gong was supported by grant K23 HL67197 from NHLBI and M.Z. Wang by grant T32 ES07069 from the National Institute of Health (Bethesda, MD, USA).

European Respiratory Journal Print ISSN 0903-1936

Online ISSN 1399-3003 
pneumonia, the $-1082 G G$ genotype is associated with increased severity and risk of sepsis and increased mortality [23, 24]. However, in studies on critical illnesses, the -1082GG genotype occurs less frequently among critically ill patients with organ failures than in healthy controls [25]. Among patients with established critical illnesses, the -1082GG genotype has been associated with significantly lower $[26,27]$ or a trend to lower severity of illness, organ dysfunction and mortality [22, 28].

The present study describes a nested case-control study of patients at risk for ARDS. The current authors investigated whether the IL-10 -1082 GA polymorphism was associated with susceptibility to developing ARDS and with mortality in ARDS.

\section{MATERIALS AND METHODS Study subjects}

Details of the study population and design have been previously described [2]. Admissions to the intensive care units (ICUs) of the Massachusetts General Hospital (Boston, MA, USA) were screened daily for study-defined clinical risk factors for ARDS, such as sepsis, pneumonia, trauma, massive transfusion of eight or more units of blood within $24 \mathrm{~h}$, or aspiration as defined previously [2]. Exclusion criteria included age $<18$ yrs, diffuse alveolar haemorrhage or chronic lung diseases and directive to withhold intubation. Patients with immunosuppression or treatment with granulocyte colony-stimulating factor were excluded. After November 2000, patients with immunosuppression from corticosteroid treatment were no longer excluded because of increasing use of steroids in sepsis.

Figure 1 displays the details of the study design and selection of cases and controls. ICU admissions with at least one defined risk factor for ARDS and no exclusion criteria were eligible for the prospective cohort and followed for development of ARDS, defined by respiratory failure requiring intubation and American European Consensus Committee (AECC) criteria, as previously described [2]. Those who fulfilled criteria for ARDS during hospitalisation were selected as cases in the case-control study. All patients who did not develop ARDS during hospitalisation and had no prior history of ARDS or prior enrolment into the study were selected as controls. The Human Subjects Committees (Boston, MA, USA) approved the study, and informed written consent was obtained from all subjects or their appropriate surrogates.

Baseline clinical information was collected on admission to ICU. Vital signs and laboratory parameters from the first $24 \mathrm{~h}$

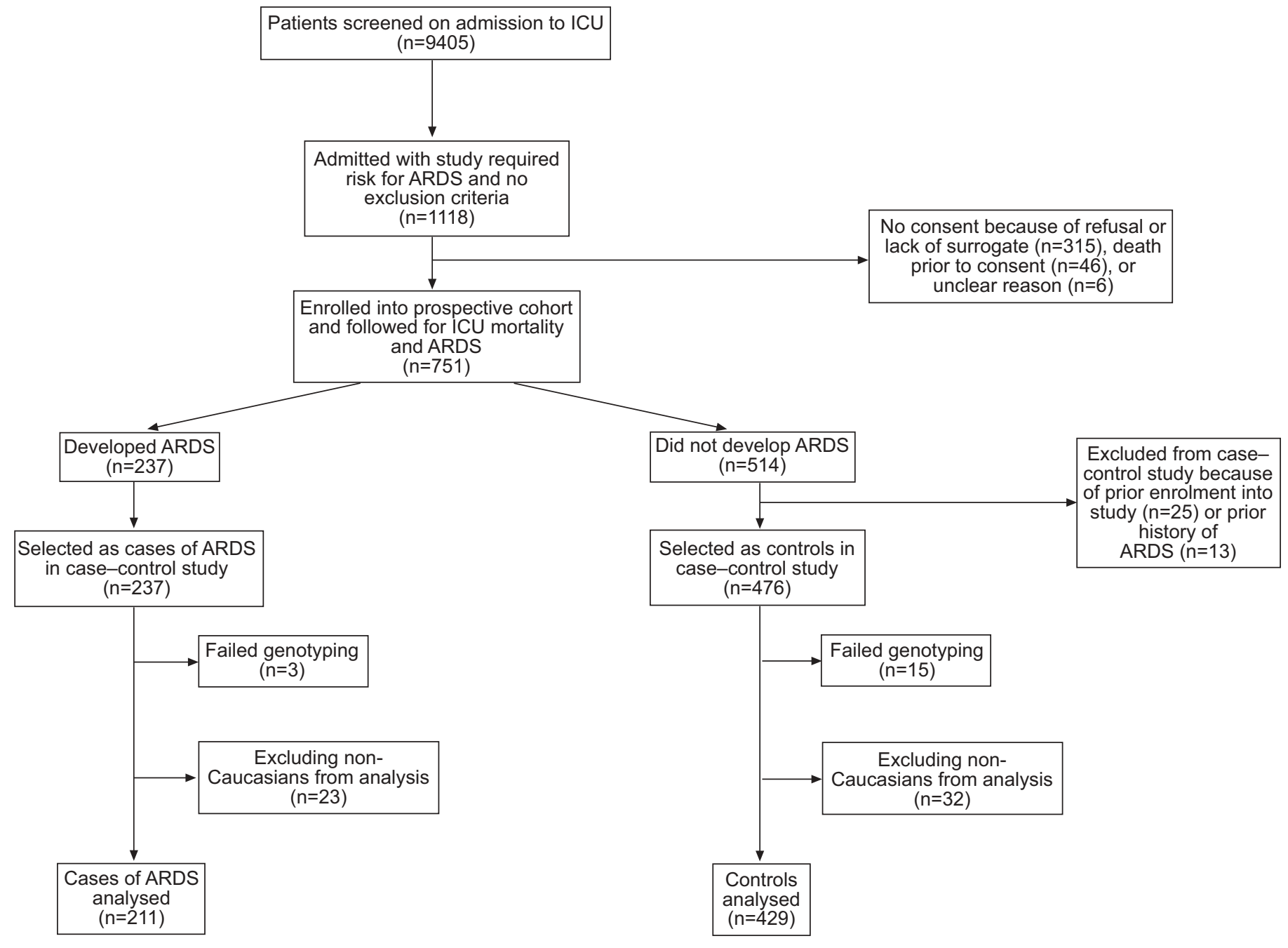

FIGURE 1. Flow diagram of study design and patient selection for the case-control study. ICU: intensive care unit; ARDS: acute respiratory distress syndrome. 
after ICU admission were collected for Acute Physiology, Age and Chronic Health Evaluation (APACHE) III study [29]. Missing physiology data occurred in $<13 \%$ of patients. Similar to the APACHE III study, missing physiology values were assumed to be within the normal range [29]. Missing nonphysiology data, such as genotype failures, history of diabetes or tobacco use, were coded as missing.

ARDS cases were followed for all-cause 60-day mortality and daily multiple organ dysfunction score (MODS) for 28 days after the development of ARDS. MODS was defined and calculated according to criteria established in Brussels [30], whereby one point is given for each organ failure. Cardiovascular failure was defined as systolic blood pressure $<90 \mathrm{mmHg}$ or need for vasopressor. Renal failure was defined as creatinine $>2.0 \mathrm{mg} \cdot \mathrm{L}^{-1}$, hepatic failure by total bilirubin $>2.0 \mathrm{mg} \cdot \mathrm{dL}^{-1}$ and haematological failure as platelets $<80,000 \cdot \mathrm{m}^{-3}$. Respiratory failure was not included in the calculation of the organ dysfunction score in the ARDS patients.

\section{Methods}

Blood $(10 \mathrm{~mL})$ was collected for DNA extraction and PCR amplification. Genotyping was performed with the Sequenom MassARRAY system [31], with a random $5 \%$ of samples repeated. Laboratory personnel and research assistants were blinded to the case-control status or genotype of the subjects.

\section{Analysis}

Continuous data are presented as mean \pm SD or as median and the 25th to 75th percentile range, depending on the distribution. Univariate analysis was performed using Fisher's exact tests, ANOVA or Wilcoxon rank sum tests as appropriate. The Kaplan-Meier curves for 60-day ARDS survival were compared using the log-rank test. Variables with p-value of $\leqslant 0.2$ on univariate analyses were studied in a backwards selection algorithm and eliminated if they did not meet a p-value of $\leqslant 0.1$. Multivariate analyses consisted of logistic regression models for ARDS and Cox proportional hazard models for mortality in ARDS and included the following: 1) the gene effect; 2) results from backwards elimination; 3) significant interactions and clinically relevant parameters, such as septic shock; and 4) APACHE III scores for the development of ARDS [32]. Only four observations for the development of ARDS and one observation for mortality in ARDS were deleted from the final multivariate models due to missing values. A HosmerLemeshow goodness-of-fit test was used to evaluate a logistic regression model fit. As per prior reports, individuals with the $-1082 \mathrm{GG}$ genotype were compared with individuals with the $-1082 A$ allele $(-1082 A A$ and $-1082 G A)$ [22, 24, 27]. Effect modification was tested with an interaction term. ARDS patients lost to follow-up, discharged home alive prior to 60 days or survived to 60 days were censored at last contact. Only APACHE III score deviated from the proportional hazard assumption, as indicated by time-varying covariate $(p=0.04)$, so the final model for ARDS survival was stratified by APACHE III score by quartiles. Daily MODS after development of ARDS was compared using mixed-effects models assuming an unstructured covariance matrix using the Proc Mixed procedure in a statistical software program. The fixed factors included the polymorphism and other potential confounders, such as age, trauma as a risk factor for ARDS, APACHE III scores, treatment with corticosteroids prior to admission, liver failure, transfusion, and septic shock. The number of days after the development of ARDS was considered to be a random factor. For patients discharged from the hospital within 28 days of ARDS, their last available organ dysfunction score prior to discharge was assigned to all subsequent days. For ARDS patients who died within 28 days

\section{TABLE 1}

Clinical risk factors for acute respiratory distress syndrome (ARDS) between cases and controls and survivors and nonsurvivors in ARDS

\begin{tabular}{|c|c|c|c|c|c|c|}
\hline \multirow[t]{2}{*}{ Risk for ARDS ${ }^{\#}$} & \multicolumn{3}{|c|}{ Development of ARDS } & \multicolumn{3}{|c|}{ Mortality in ARDS } \\
\hline & Controls & Cases $^{+}$ & p-value & Survivors $^{\S}$ & Nonsurvivors ${ }^{f}$ & p-value \\
\hline Sepsis syndrome & $163(38)$ & $67(32)$ & 0.1 & $44(39)$ & $23(23)$ & 0.02 \\
\hline Pneumonia source & $85(20)$ & $52(25)$ & $<0.001$ & $33(29)$ & $19(19)$ & 0.6 \\
\hline Extrapulmonary source & $78(18)$ & $15(7)$ & $<0.001$ & $11(10)$ & $4(4)$ & \\
\hline Septic shock & $191(45)$ & $114(54)$ & 0.03 & $51(45)$ & $63(64)$ & 0.006 \\
\hline Pneumonia source & $85(20)$ & $78(37)$ & $<0.001$ & $35(31)$ & $43(44)$ & ( \\
\hline Extrapulmonary source & $106(25)$ & $36(17)$ & $<0.001$ & $16(14)$ & $20(20)$ & $>0.9$ \\
\hline Trauma & $38(9.0)$ & $9(4)$ & 0.04 & $8(7)$ & $1(1)$ & 0.04 \\
\hline Multiple transfusions & $50(12)$ & $26(12)$ & 0.8 & $13(12)$ & $13(13)$ & 0.8 \\
\hline Aspiration & $32(7)$ & $24(11)$ & 0.1 & $13(12)$ & $11(11)$ & $>0.9$ \\
\hline One or more risk for ARDS & $48(11)$ & $29(14)$ & 0.3 & $16(14)$ & $13(13)$ & $>0.9$ \\
\hline Direct pulmonary injury & $204(48)$ & $143(68)$ & $<0.001$ & $78(69)$ & $65(66)$ & 0.8 \\
\hline Indirect pulmonary injury & $225(52)$ & $68(32)$ & $<0.001$ & $35(31)$ & $33(34)$ & \\
\hline
\end{tabular}

Data are presented as $n$ (\%), unless otherwise stated. * : numbers of controls and cases with each risk add up to $>640$ patients because of multiple risks in 75 patients; ${ }^{\natural}$ $n=429 ;^{+}: n=211 ;{ }^{\S}: n=113 ;{ }^{f}: n=98 ;{ }^{\# \#}$ : pneumonia, aspiration or pulmonary contusions were categorised as direct pulmonary injury; ${ }^{\bullet}{ }^{\prime}$ : sepsis from an extrapulmonary source, trauma without pulmonary contusions and multiple transfusions were categorised as indirect pulmonary injury; patients ( $\mathrm{n}=69$ ) with both direct and indirect pulmonary injuries were considered to have direct pulmonary injury. 
of ARDS, the maximal score was assigned to all days subsequent to death. A p-value of 0.05 was considered statistically significant.

In the initial study design, assuming an $\alpha$-error of 0.01 for multiple comparisons, $80 \%$ power and allele frequency of 0.51 for $-1082 G$, a study with 560 cases and 1,120 controls would have a minimum detectable odds ratio (OR) for the development of ARDS of 1.43. Given the actual study size of 211 cases, 429 controls and $\alpha$-error of 0.05 without adjusting for multiple comparisons, the minimum detectable OR would be 0.62 or 1.61 for ARDS and 0.45 or 2.2 for mortality in ARDS.

\section{RESULTS}

\section{Patient population}

The case-control study consisted of 237 ARDS cases and 477 controls recruited between September 9, 1999 and October 15, 2002 (fig. 1). A total of 18 (3\%) patients failed genotyping. As $92 \%$ of the remaining patients were Caucasians, analyses were restricted to the 211 Caucasian cases and 429 Caucasian controls.

Clinical risk factors for ARDS and baseline characteristics on admission to the ICU are shown in tables 1 and 2. Variables in the final model for development of ARDS included direct pulmonary injury $(\mathrm{p}<0.001)$, trauma $(\mathrm{p}<0.001)$, age $(p<0.001)$, female sex $(p=0.04)$, diabetes $(p=0.001)$, platelets $\leqslant 80,000 \cdot \mathrm{mm}^{-1}(p=0.002)$, blood transfusion $(p<0.001)$, septic shock $(p=0.2)$ and APACHE III score $(p=0.4)$. The HosmerLemeshow statistic for the final model was 6.1 with eight degrees of freedom $(p=0.6)$, indicating no significant lack of fit of the model.

\section{Genotype analyses and development of ARDS}

The allele frequency for the $-1082 G$ allele was 0.45 . There was no discrepancy on repeat genotyping. Genotype frequency among cases and controls is shown in table 3. Among the controls, the genotype frequency deviated from that predicted by the Hardy-Weinberg equilibrium $(p=0.04)$. Among all patients in the study, the APACHE III score on admission did not differ significantly by the $-1082 G A$ genotype $(p=0.8$; fig. 2a). The $-1082 G G$ genotype was associated with development of ARDS on the final multivariate analysis (crude OR $1.1 ; 95 \%$ confidence interval (CI; 0.74-1.6); adjusted OR 22; 95\% CI (3.9-126)), but only in the presence of an interaction term between the $I L-10-1082 G G$ genotype and age $(p<0.001$ for main effect and for interaction). Adjusting for any of the other covariates in the model changed the crude estimate $<5 \%$ with no qualitative change in significance. This interaction between age and the -1082GG genotype was examined further after stratifying by age according to quartiles (table 3; fig. 3). The -1082GG genotype was significantly associated with an increased odds of developing ARDS among individuals $<52$ yrs of age (ORcrude 3.9; 95\% CI (1.8-8.6); ORadj 5.1; 95\% CI (2.0-13); p<0.001). However, when patients aged $\geqslant 52$ yrs were examined together, the -1082GG genotype appeared protective against ARDS (ORcrude 0.70; 95\% CI (0.44-1.1); ORad 0.59 ; 95\% CI (0.350.99); $\mathrm{p}=0.04)$.

\section{TABLE 2 Baseline characteristics between cases of acute respiratory distress syndrome (ARDS) and controls and survivors and} nonsurvivors in ARDS

\begin{tabular}{|c|c|c|c|c|c|c|}
\hline & \multicolumn{3}{|c|}{ Development of ARDS } & \multicolumn{3}{|c|}{ Mortality in ARDS } \\
\hline Age yrs & $69(18-94)$ & $65(18-97)$ & 0.04 & $57(18-89)$ & $73(22-97)$ & $<0.001$ \\
\hline APACHE III ${ }^{f}$ & $64(14-130)$ & $68(8-136)$ & 0.09 & $69(8-115)$ & $88(29-150)$ & $<0.001$ \\
\hline Diabetes $^{\# \#}$ & $114(27)$ & $33(16)$ & 0.002 & $19(17)$ & $14(14)$ & 0.7 \\
\hline Tobacco abuse" & $211(49)$ & $104(49)$ & 0.4 & $56(50)$ & $48(49)$ & 0.9 \\
\hline Chronic liver disease ${ }^{\# \#}$ & $18(4)$ & $12(6)$ & 0.7 & $4(4)$ & $8(8)$ & 0.2 \\
\hline End-stage renal disease & $22(5)$ & $6(3)$ & 0.2 & $2(2)$ & $4(4)$ & 0.4 \\
\hline History of steroid use & 37 (9) & $20(9)$ & 0.7 & $6(5)$ & $14(14)$ & 0.03 \\
\hline Transfusion of PRBC & $213(50)$ & $133(63)$ & 0.001 & $64(57)$ & $69(70)$ & 0.03 \\
\hline $\begin{array}{l}\text { Number of PRBC } \\
\text { transfused }\end{array}$ & $0(0-74)$ & $2(0-63)$ & 0.005 & $1(0-31)$ & $2(0-63)$ & 0.02 \\
\hline $\begin{array}{l}\text { Haematological failure } \\
\text { platelets } \leqslant 80000 \cdot \mathrm{mm}^{-1}\end{array}$ & $58(14)$ & $47(22)$ & 0.006 & $20(18)$ & $27(28)$ & 0.1 \\
\hline
\end{tabular}

Data are presented as $\mathrm{n}(\%)$ or median (range), unless otherwise stated. APACHE: Acute Physiology, Age and Chronic Health Evaluation; PRBC: packed red blood cells BP: blood pressure. ${ }^{\#}: n=429 ;{ }^{\bullet}: n=211 ;{ }^{+}: n=113 ;{ }^{\S}: n=98 ;{ }^{f}:$ for development of ARDS, APACHE III physiology score for cases and controls were calculated without the arterial oxygen tension/inspiratory oxygen fraction component; for survivors and nonsurvivors in ARDS, the APACHE III physiology score was calculated with all components; ${ }^{\# \#}$ : chronic health information was missing in one case and two controls; " ": tobacco history was missing in 56 (26\%) cases and 97 (22\%) controls. 


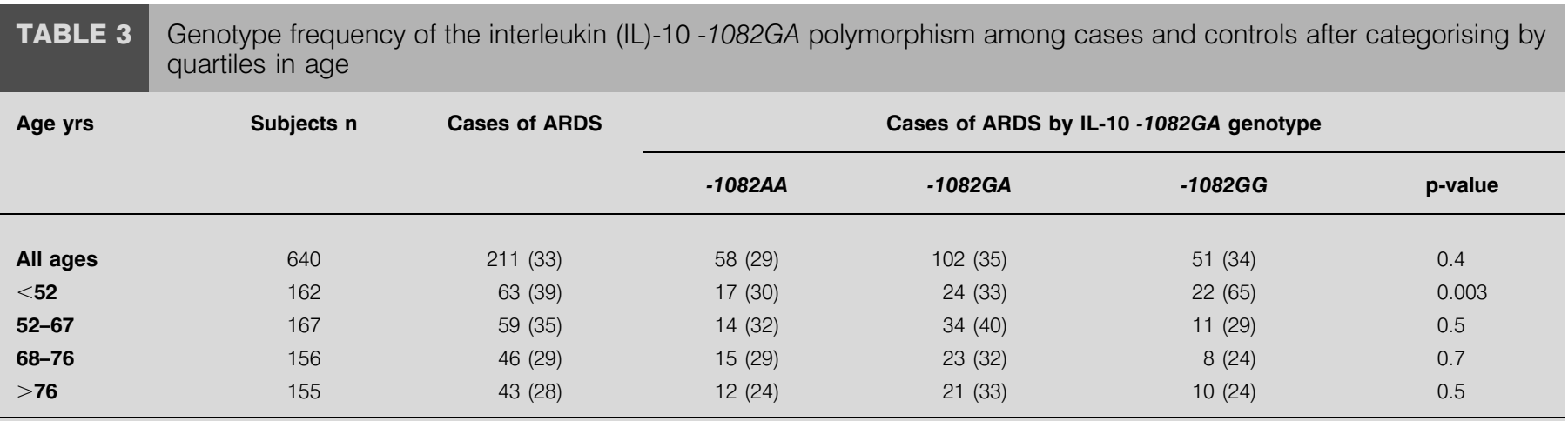

Data are presented as $n$ or $n(\%)$, unless otherwise stated. ARDS: acute respiratory distress syndrome.
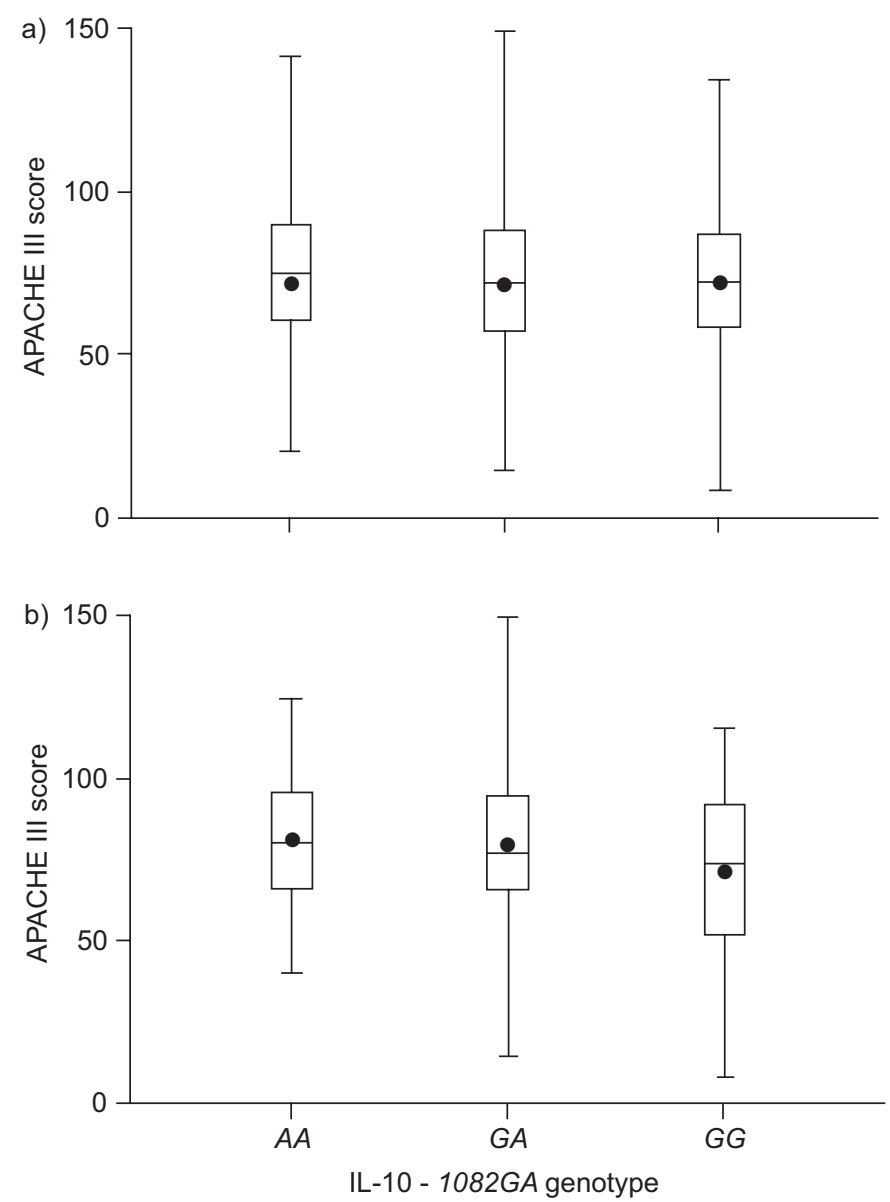

FIGURE 2. Acute Physiology, Age and Chronic Health Evaluation (APACHE) III score on admission to the intensive care unit by genotype for the interleukin (IL)-10 -1082GA polymorphism among a) 640 cases and controls (AA: $\mathrm{n}=200 ; \mathrm{GA}: \mathrm{n}=292$; GG: $n=148 ; \mathrm{p}=0.8$ ) and $b$ ) the 211 ARDS cases (AA: $n=58 ; G A: n=102 ; G G$ : $\mathrm{n}=51 ; \mathrm{p}=0.008)$. The box denotes the interquartile range (25-75\%) and the horizontal line indicates the median. Error bars indicate the 95\% confidence intervals.

\section{IL-10 -1082GA polymorphism and outcomes in ARDS}

The 60-day mortality was $46 \%$ (98 out of 211) for ARDS cases. Clinical risks for ARDS and baseline characteristics between survivors and nonsurvivors of ARDS are shown in tables 1 and
2. Significant predictors in the final model for mortality in ARDS included age $(p<0.001)$, APACHE III score $(p=0.001)$, trauma $(p=0.08)$, systolic blood pressure $<90 \mathrm{mmHg}(\mathrm{p}=0.02)$, alcohol abuse $(p=0.03)$ and blood transfusion $(p=0.001)$. No significant interactions were found.

Compared with ARDS patients with $-1082 A A$ and $-1082 G A$ genotypes, ARDS patients with the $-1082 G G$ genotype had lower APACHE III scores on admission to the ICU $(70.7 \pm 25.3$ versus $81.0 \pm 23.2 ; \mathrm{p}=0.008$; fig. $2 \mathrm{~b}$ ) and higher ventilator-free days (median (interquartile range) $3(0-12)$ versus $0(0-10)$ ), although the latter was not statistically significant $(\mathrm{p}=0.3)$. After development of ARDS, the -1082GG ARDS cases had lower daily organ dysfunction scores over time than the $-1082 \mathrm{~A}$ allele carriers $(\mathrm{p}=0.03$; fig. 4). The 60-day mortality among the ARDS cases varied significantly with the $-1082 G A$ genotype (fig. 5). In comparison to the $-1082 A$ carriers, the $-1082 G G$ genotype was associated with decreased 60-day mortality in ARDS on crude analyses (hazard ratio (HR) 0.43 ; $95 \%$ CI $(0.25$ $0.76) ; p=0.004)$ with the difference in mortality occurring early in the course of ARDS (fig. 5). On multivariate analysis after controlling for potential confounders and stratifying by APACHE III score on admission, the association between $-1082 G G$ genotype and ARDS mortality remained significant (HRadj 0.55; 95\% CI (0.31-0.99); $\mathrm{p}<0.05)$.

\section{DISCUSSION}

In a study of ICU patients with clearly defined ARDS risk factors, the current authors found that the -1082GG genotype was associated with ARDS, but only in the presence of a significant interaction between the $-1082 G G$ genotype and age. Among patients with ARDS, significant associations were found between the IL-10 -1082GG genotype and lower severity of illness on presentation, lower daily organ dysfunction and lower mortality.

The present study has a number of strengths. First, the prospective determination of ARDS using the AECC definition helps minimise phenotype misclassification. Secondly, clearly defined at-risk controls were used. Using critically ill controls who have the opportunity to develop the outcome is more clinically relevant than using healthy individuals. This also reduces any confounding from any possible association between the gene and the risk condition, such as sepsis or pneumonia. 


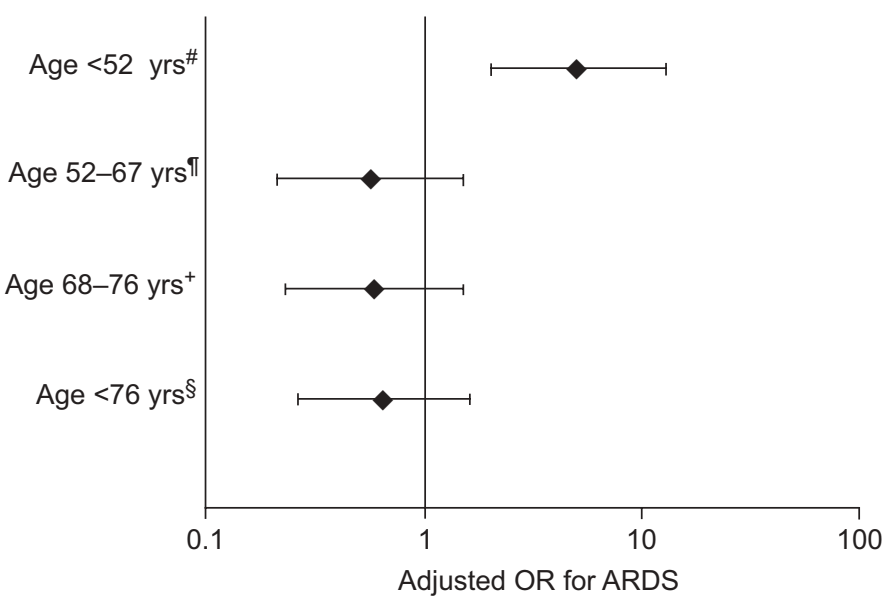

FIGURE 3. Odds ratio (OR) for the development of acute respiratory distress syndrome (ARDS) among individuals homozygous for -1082G allele (1082GG) compared with carriers of the $-1082 \mathrm{~A}$ allele after stratifying by age quartiles. OR were adjusted for direct pulmonary injury, septic shock, trauma, female sex, haematological failure (platelet $<80,000 \cdot \mathrm{mm}^{-1}$ ), transfusion of red cells and Acute Physiology, Age and Chronic Health Evaluation III (without the arterial oxygen tension/inspiratory oxygen fraction component). There was significant interaction by age on the association between the interleukin-10 -1082GG genotype and development of ARDS $(p<0.001) .{ }^{*}: n=161 ;{ }^{\circ}: n=165 ;{ }^{+}: n=155 ;{ }^{s}: n=154$.

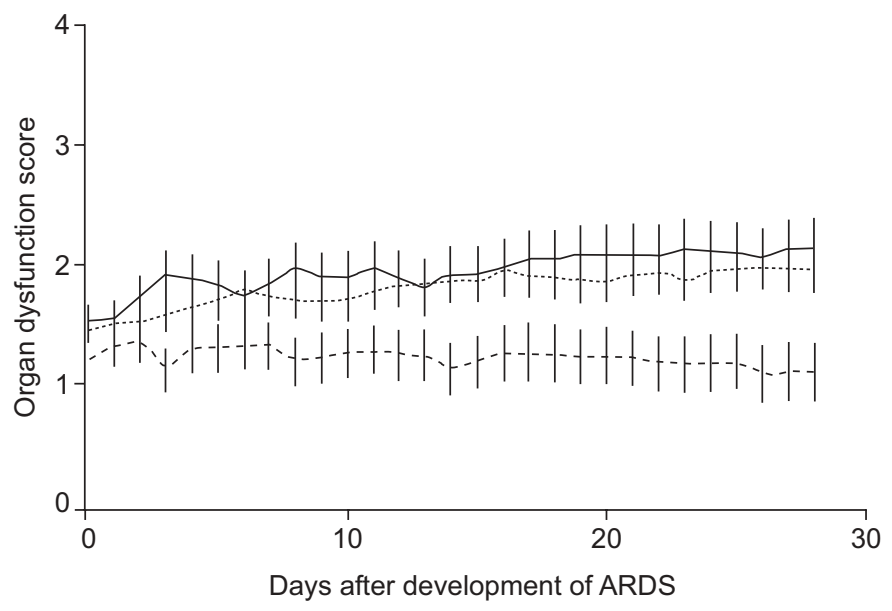

FIGURE 4. Daily Brussels multiple organ dysfunction score for 28 days after development of acute respiratory distress syndrome (ARDS) according to interleukin-10 -1082GA genotype ( $\cdots)$ for the 211 ARDS cases. In comparison with the $-1082 A$ carriers $(-1082 A A(-)$ and $-1082 \mathrm{GA}(--))$, ARDS patients with the $-1082 G G$ genotype had less organ failure over the course of their ARDS $(p=0.03)$

The significance of the interaction between age and the $-1082 G G$ genotype on the risk of ARDS is unclear. It is possible that the relative pro- versus anti-inflammatory response to infection and injury differs with age. Elderly patients have elevated levels of circulating pro-inflammatory cytokines, such as IL-6 and TNF- $\alpha$ at baseline and in sepsis [32-34]. In a population prone to a higher inflammatory state, a genotype associated with high anti-inflammatory response may be protective against development of ARDS, whereas in another group less prone to inflammation, the effect may be different. However, given that the baseline inflammatory state of the

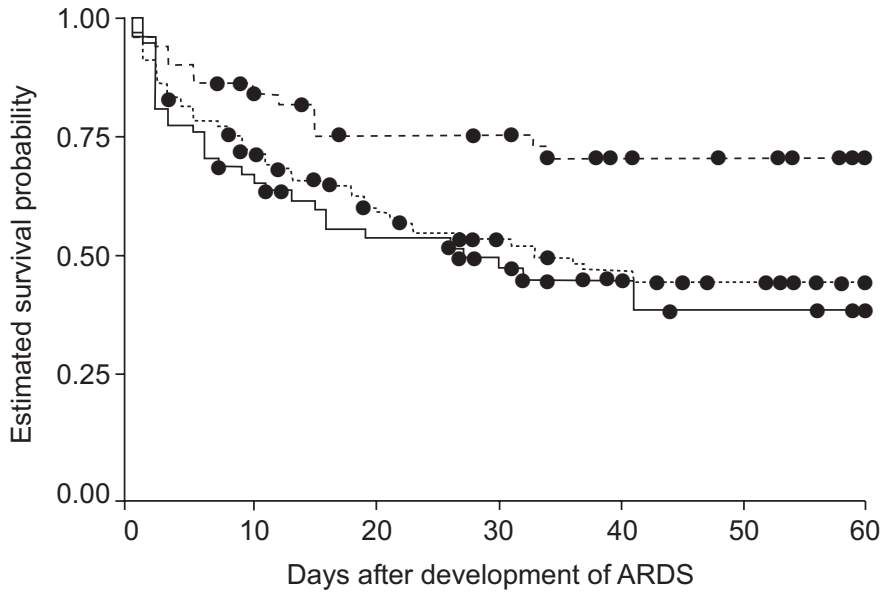

FIGURE 5. Kaplan-Meier 60-day survival curves in acute respiratory distress syndrome (ARDS) according to the interleukin-10 -1082GA genotype. Compared with -1082AA ( $\longrightarrow$ and -1082GA ( $\cdots)$ genotypes. ARDS patients with the -1082GG genotype (- - ) had better survival $(p=0.004)$. $\bullet$ : censored.

patients could not be examined here, this finding should be used for hypothesis generation. Additional studies will be needed to define the biological interaction between the $-1082 G G$ genotype and age in ARDS.

The current finding that the high IL-10-producing -1082GG genotype is protective against mortality and organ failure in ARDS is consistent with the role of intense inflammation in acute lung injury. Other studies have found significant or nonsignificant associations between the $I L-10-1082 \mathrm{G}$ allele and decreased organ failure, severity of illness or mortality in critically ill patients [22, 26-28]. However, in noncritically ill patients with pneumonia, the $-1082 G G$ genotype was associated with increased severity of sepsis and mortality [23, 24]. The different patient populations studied may explain this discrepancy. It is increasingly recognised that the overall balance between pro-inflammatory and anti-inflammatory responses is important in the response to injury and in ARDS [35-37]. In patients with pneumonia, a genotype associated with high IL-10 production may lead to more immunosuppression, resulting in more severe disease burden and outcomes. In contrast, the present findings were in ARDS patients. As intense inflammation is a key feature in the pathogenesis of ARDS, the present authors may have selected for patients with a high pro-inflammatory condition. In such cases, a genotype associated with high IL-10 may have a beneficial modulating effect. Indeed, other studies that have found a protective effect of the $-1082 \mathrm{G}$ allele were carried out in critically ill patients or in meningococcaemia in which an intense pro-inflammatory response is well recognised [26, 27]. Given the high frequency of the $-1082 \mathrm{G}$ allele in the population, it is reasonable that the high IL-10-producing -1082GG genotype may not be universally detrimental. Thus, it is important to note that the current findings may not be applicable to non-ARDS patients.

It is also interesting to note that the mortality difference among ARDS patients with the $-1082 G G$ genotype occurs early in the course of ARDS, reaching a plateau in the later course of 
ARDS. The potential benefit of a high anti-inflammatory genotype may not be the same through all stages of ARDS. Certainly the cause of mortality and the balance between proand anti-inflammatory responses in critical illnesses differs over time [38-40].

Among the controls in the present study, the genotype frequency of the $-1082 G A$ polymorphism deviated from that predicted by the Hardy-Weinberg equilibrium. This is unlikely to be due to genotyping error as repeat genotyping in a random subset of patients revealed no discrepancy. However, since the controls were critically ill patients with organ failure, it is possible that deviation from Hardy-Weinberg occurred because of a possible association between the $-1082 \mathrm{GA}$ polymorphism and the critical illness requiring ICU admission, as has been reported by other groups $[25,41]$.

The association between the -1082GG genotype, lower APACHE III score and lower ARDS mortality is unlikely to be due to the increased odds of younger patients with this genotype to develop ARDS. The association between the -1082GG genotype and APACHE score in ARDS was still significant even after age was removed from the score $(\mathrm{p}<0.03)$. The association between the $-1082 \mathrm{GG}$ genotype and decreased mortality remained even after restricting the analyses to those patients $<52$ yrs of age (HR 0.083; 95\% CI (0.01-0.68); $\mathrm{p}=0.006$; data not shown).

The association found between the IL-10 -1082GA polymorphism and ARDS mortality is unlikely to be due to type I error. The polymorphism was chosen a priori based upon previous studies supporting its role in critical illness. The results are consistent with previous reports on organ failure and mortality in critical illness. Additionally, the results are supported by results of secondary outcomes, such as severity of illness on admission and organ failure after development of ARDS. Nevertheless, as is true of all genetic association studies, the current findings will need to be confirmed in other populations.

The present authors acknowledge that there are some limitations to the study. Only one polymorphism was examined in the $I L-10$ gene and $-1082 G A$ polymorphism is known to be linked to other functional polymorphisms on the $I L-10$ gene in other disease conditions [42-44]. Thus, the possibility that the association found in the current study is due to linkage disequilibrium between the $-1082 \mathrm{G}$ allele and the disease locus cannot be excluded. A haplotype approach would be better to examine this possibility. In addition, the functional significance of the $-1082 G A$ polymorphism cannot be confirmed in the current study. Due to the study design, the results may not be generalisable to the community setting, immunocompromised hosts or healthy patients without risk factors for ARDS, or with different clinical risks for ARDS. In addition, the analyses were restricted to Caucasians, which reduces the possibility of confounding from ethnicity [45], but it does not permit extrapolation of the results to other ethnic groups.

In conclusion, the present study reports an interaction between the interleukin-10 -1082GG genotype and age on the development of acute respiratory distress syndrome. Among cases of acute respiratory distress syndrome, the interleukin-10-1082GG genotype was associated with decreased severity of illness, decreased organ dysfunction and decreased mortality in acute respiratory distress syndrome. Additional studies are needed to confirm these findings in other populations with other risk factors.

\section{ACKNOWLEDGEMENTS}

The authors would like to thank W-L. Zhang, K. McCoy, T. McCabe, C. Schwartzenburg, B. Bean and L. Lineback for patient recruitment; A. Solomon, A. Shafer and L. Shimada for research support; L. Su for laboratory expertise; J. Frelich, M. Chertok, S. Mucci, R. Rivera for data management; and P.D. Boyce for assistance in radiological interpretation.

\section{REFERENCES}

1 Hudson LD, Milberg JA, Anardi D, Maunder RJ. Clinical risks for development of the acute respiratory distress syndrome. Am J Respir Crit Care Med 1995; 151: 293-301.

2 Gong MN, Thompson BT, Pothier L, Boyce P, Christiani DC. Predictors for the development of and mortality in ARDS: potential role of red cell transfusion. Crit Care Med 2005; 33: 1191-1198.

3 Marshall RP, Webb S, Bellingan GJ, et al. Angiotensin converting enzyme insertion/deletion polymorphism is associated with susceptibility and outcome in acute respiratory distress syndrome. Am J Respir Crit Care Med 2002; 166: 646-650.

4 Quasney MW, Waterer GW, Dahmer MK, et al. Association between surfactant protein $\mathrm{B}+1580$ polymorphism and the risk of respiratory failure in adults with communityacquired pneumonia. Crit Care Med 2004; 32: 1115-1119.

5 Garred P, Strom JJ, Quist L, Taaning E, Madsen HO. Association of mannose-binding lectin polymorphisms with sepsis and fatal outcome, in patients with systemic inflammatory response syndrome. J Infect Dis 2003; 188: 1394-1403.

6 Oberholzer A, Oberholzer C, Moldawer LL. Interleukin-10: a complex role in the pathogenesis of sepsis syndromes and its potential as an anti-inflammatory drug. Crit Care Med 2002; 30: Suppl. 1, S58-S63.

7 Latifi SQ, O'Riordan MA, Levine AD. Interleukin-10 controls the onset of irreversible septic shock. Infect Immun 2002; 70: 4441-4446.

8 Walley KR, Lukacs NW, Standiford TJ, Strieter RM, Kunkel SL. Balance of inflammatory cytokines related to severity and mortality of murine sepsis. Infect Immun 1996; 64: 4733-4738.

9 Steinhauser ML, Hogaboam CM, Kunkel SL, Lukacs NW, Strieter RM, Standiford TJ. IL-10 is a major mediator of sepsis-induced impairment in lung antibacterial host defense. J Immunol 1999; 162: 392-399.

10 Marchant A, Deviere J, Byl B, De Groote D, Vincent JL, Goldman M. Interleukin-10 production during septicaemia. Lancet 1994; 343: 707-708.

11 Neidhardt R, Keel M, Steckholzer U, et al. Relationship of interleukin-10 plasma levels to severity of injury and clinical outcome in injured patients. J Trauma 1997; 42: 863-870.

12 Igonin AA, Armstrong VW, Shipkova M, Lazareva NB, Kukes VG, Oellerich M. Circulating cytokines as markers 
of systemic inflammatory response in severe communityacquired pneumonia. Clin Biochem 2004; 37: 204-209.

13 Monneret G, Finck ME, Venet F, et al. The anti-inflammatory response dominates after septic shock: association of low monocyte HLA-DR expression and high interleukin-10 concentration. Immunol Lett 2004; 95: 193-198.

14 Simmons EM, Himmelfarb J, Sezer MT, et al. Plasma cytokine levels predict mortality in patients with acute renal failure. Kidney Int 2004; 65: 1357-1365.

15 Armstrong L, Millar AB. Relative production of tumor necrosis factor- $\alpha$ and interleukin-10 in adult respiratory distress syndrome. Thorax 1997; 52: 442-446.

16 Parsons PE, Moss M, Vannice JL, Moore EE, Moore FA, Repine JE. Circulating IL-1ra and IL-10 levels are increased but do not predict the development of acute respiratory distress syndrome in at-risk patients. Am J Respir Crit Care Med 1997; 155: 1469-1473.

17 Donnelly SC, Strieter RM, Reid PT, et al. The association between mortality rates and decreased concentrations of interleukin-10 and interleukin-1 receptor antagonist in the lung fluids of patients with the adult respiratory distress syndrome. Ann Intern Med 1996; 125: 191-196.

18 Westendorp RG, Langermans JA, Huizinga TW, et al. Genetic influence on cytokine production and fatal meningococcal disease. Lancet 1997; 349: 170-173.

19 Reuss E, Fimmers R, Kruger A, Becker C, Rittner C, Hohler T. Differential regulation of interleukin-10 production by genetic and environmental factors-a twin study. Genes Immun 2002; 3: 407-413.

20 Turner DM, Williams DM, Sankaran D, Lazarus M, Sinnott PJ, Hutchinson IV. An investigation of polymorphism in the interleukin-10 gene promoter. Eur J Immunogenet 1997; 24: 1-8.

21 Suarez A, Castro P, Alonso R, Mozo L, Gutierrez C. Interindividual variations in constitutive interleukin-10 messenger RNA and protein levels and their association with genetic polymorphisms. Transplantation 2003; 75: 711-717.

22 Galley HF, Lowe PR, Carmichael RL, Webster NR. Genotype and interleukin-10 responses after cardiopulmonary bypass. Br J Anaesth 2003; 91: 424-426.

23 Gallagher PM, Lowe G, Fitzgerald T, et al. Association of IL-10 polymorphism with severity of illness in community acquired pneumonia. Thorax 2003; 58: 154-156.

24 Schaaf BM, Boehmke F, Esnaashari H, et al. Pneumococcal septic shock is associated with the interleukin-10-1082 gene promoter polymorphism. Am J Respir Crit Care Med 2003; 168: 476-480.

25 Reid CL, Perrey C, Pravica V, Hutchinson IV, Campbell IT. Genetic variation in proinflammatory and anti-inflammatory cytokine production in multiple organ dysfunction syndrome. Crit Care Med 2002; 30: 2216-2221.

26 Balding J, Healy CM, Livingstone WJ, et al. Genomic polymorphic profiles in an Irish population with meningococcaemia: is it possible to predict severity and outcome of disease? Genes Immun 2003; 4: 533-540.

27 Jaber BL, Rao M, Guo D, et al. Cytokine gene promoter polymorphisms and mortality in acute renal failure. Cytokine 2004; 25: 212-219.

28 Schroder O, Laun RA, Held B, Ekkernkamp A, Schulte KM. Association of interleukin-10 promoter polymorphism with the incidence of multiple organ dysfunction following major trauma: results of a prospective pilot study. Shock 2004; 21: 306-310.

29 Knaus WA, Wagner DP, Draper EA, et al. The APACHE III prognostic system. Risk prediction of hospital mortality for critically ill hospitalized adults. Chest 1991; 100: 1619-1636.

30 Bernard GR, Doig G, Hudson L. Quantification of organ failure for clinical trials and clinical practice. Am J Respir Crit Care Med 1995; 151: A323.

31 Jurinke C, van den Boom D, Cantor CR, et al. The use of MassARRAY technology for high throughput genotyping. Adv Biochem Eng Biotechnol 2002; 77: 57-74.

32 Hosmer DW, Lemeshow S. Assessing the fit of model. In: Applied Logistic Regression. 2nd Edn. USA, WileyInterscience Publication, 2000; pp. 160-164.

33 Ershler WB, Keller ET. Age-associated increased interleukin-6 gene expression, late life disease and frailty. Annu Rev Med. 2000; 51: 245-270.

34 Marik PE, Zalonga GP, Norasept II Study Investigators. The effect of aging on circulating levels of pro-inflammatory cytokines during septic shock. J Am Geriatric Soc 2001; 49: 5-9.

35 Bruunsgaard H, Pedersen BK. Age-related inflammatory cytokines and disease. Immunol Allergy Clin North Am 2003; 23: 15-39.

36 Gogos CA, Drosou E. Pro- versus anti-inflammatory cytokine profile in patients with severe sepsis: a marker for prognosis and future therapeutic options. J Infect Dis 2000; 181: 176-180.

37 Goodman RB, Pugin J, Lee JS, Matthay MA. Cytokinemediated inflammation in acute lung injury. Cytokine Growth Factor Rev 2003; 14: 523-535.

38 Zanotti S, Kumar A, Kumar A. Cytokine modulation in sepsis and septic shock. Expert Opin Investig Drugs 2002; 11: 1061-1075.

39 Montgomery AB, Stager MA, Carrico CJ, Hudson LD. Causes of mortality in patients with adult respiratory distress syndrome. Am Rev Respir Dis 1985; 132: 485-489.

40 Bone RC. Sir Isaac Newton, sepsis, SIRS, and CARS. Crit Care Med 1996; 24: 1125-1128.

41 Park WY, Goodman RB, Steinberg KP, et al. Cytokine balance in the lungs of patients with acute respiratory distress syndrome. Am J Respir Crit Care Med 2001; 164: 1896-1903.

42 Wacholder S, Rothman N, Caporaso N. Population stratification in epidemiologic studies of common genetic variants and cancer: quantification of bias. J Natl Cancer Inst 2000; 92: 1151-1158.

43 Lin MT, Storer B, Martin PJ, et al. Relation of an interleukin-10 promoter polymorphism to graft-versus-host disease and survival after hematopoietic-cell transplantation. N Engl J Med 2003; 349: 2201-2210.

44 Ma SL, Tang NL, Lam LC, Chiu HF. The association between promoter polymorphism of the interleukin-10 gene and Alzheimer's disease. Neurobiol Aging 2005; 26: 1005-1010.

45 Lowe PR, Galley HF, Abdel-Fattah A, Webster NR. Influence of interleukin-10 polymorphisms on interleukin10 expression and survival in critically ill patients. Crit Care Med 2003; 31: 34-38. 